\title{
Time to Integrate Prospective Memory Improvement Strategies in Cognitive Remediation Program for Schizophrenia?
}

\author{
Devvarta Kumar · Jagadisha Thirthalli
}

Received: 4 October 2021 / Accepted: 4 October 2021 / Published online: 13 October 2021

(C) The Author(s), under exclusive licence to Springer Nature India Private Limited 2021

Prospective memory (ProM) refers to memory for future intentions. It involves: (a) formation of an intention to execute a task at a specific point in the future, (b) retention of the intention while engaging in other unrelated tasks till the juncture to execute the intention is reached, and (c) execution of the intention at the right juncture $[1,2]$. The specific point for the execution of an intention can be the end of an activity (e.g., deciding to attach a document with an email after writing the content of the mail), onset of a particular event (e.g., an intention to inform a colleague about a plan when you meet him in the departmental meeting the next day), or a specific time (e.g., deciding in the morning to make a phone call to a friend at $8 \mathrm{PM}$ ). Thus, many problems that we encounter in our daily life, such as missing an appointment with someone, realizing later that something important had to be done, and spoiling the food on the stove due to the failure to turn it off at the appropriate time could be due to ProM failures. Given its critical role in activities

\section{Kumar $(\bowtie)$}

Department of Clinical Psychology, National Institute of Mental Health and Neurosciences (NIMHANS)

Bengaluru, Bengaluru 560029, India

e-mail: devvarta.k@nimhans.ac.in

\section{J. Thirthalli}

Department of Psychiatry, National Institute of Mental Health and Neurosciences (NIMHANS) Bengaluru,

Bengaluru, India that require self-initiation and execution, ProM becomes crucial for independent living. No wonder, on a typical day, people invest a lot of time in thinking and planning the tasks that involve ProM [3].

Successful execution of a ProM task depends on a myriad of factors such as the support of the cognitive functions required for encoding, maintenance, and retrieval of an intention; motivation of the individual to execute the task; perceived importance of the task, and so on $[4,5]$. Therefore, though all the ProM failures result in the non-execution of the intended task, the underlying mechanisms of the failures can differ. For example, failure can happen due to poor encoding of the intention, inability to identify the cue even if the memory for the intention was intact, failing to respond despite the cue being accurately recognized, and disinterest in executing the intention.

\section{ProM Deficits in Schizophrenia}

There are compelling reasons to focus on ProM impairment in schizophrenia. Many difficulties faced by the patients with schizophrenia, such as missing appointments with clinicians or irregular medication intake, could be partly due to ProM failures. Hence, if the patients are helped improve ProM, it can positively impact their everyday functioning, including treatment adherence $[6,7]$. Another reason to study ProM in schizophrenia is the neural substrate of this memory 
system. Frontal lobes play a crucial role in ProM [8, 9], and impaired frontal lobe functioning is well-known in schizophrenia [10, 11]. Therefore, impaired frontal lobe functioning may mediate ProM failures in this condition.

Patients with schizophrenia perform poorly on ProM tasks and this is largely independent of the severity of psychopathology [12] as well as the chronicity of illness [13, 14]. Further, studies on drug-naïve patients indicate that ProM deficits cannot be attributed only to the effects of medications $[12,15,16]$. Moreover, ProM impairments in schizophrenia cannot be considered solely as the result of impairment in other cognitive abilities such as retrospective memory or executive functions. Though these impairments may play a role in ProM deficits in schizophrenia, it may be noted that even after controlling their effects, ProM deficits remain, albeit to a lesser degree [17]. Besides, healthy first-degree relatives of patients with schizophrenia also display ProM impairments [18, 19]. Taken together, these findings suggest that ProM impairment could be a trait marker in schizophrenia. More importantly, ProM impairment in schizophrenia can significantly influence patients' everyday functioning (including medication adherence) and community living skills [14]. For instance, performance on ProM measures predicts the functional community living skills of patients with schizophrenia even after controlling for the effects of clinical, cognitive and, socio-demographic variables $[20,21]$. Likewise, the level of performance on the tests of ProM predicts the performance on a standardized measure of medication management skills (the Medication Management Ability Assessment Manual; MMAA; [22]). It remains so even when the measures of other cognitive functions such as attention, setshifting, and verbal learning and memory are accounted for [7].

Thus, it is evident that ProM impairment is one of the core cognitive deficits in schizophrenia, and it is a critical determinant of disability observed in patients. Therefore, ProM improvement strategies must be made an integral component of the cognitive remediation program for patients with schizophrenia.

\section{Remedial Strategies for ProM Deficits}

Of late, various approaches to improve ProM in patients with schizophrenia have been researched. Cognitive remediation strategies focus on one or more stages of ProM, that is (a) to help the individual strengthen the encoding of intention, (b) to assist in maintaining the intention during the interval between intention formation and realization, and (c) to reinforce the cue-detection process so that the task is executed at the right juncture. Patients with schizophrenia may have a problem forming a representation of the cue-response pairing or retrieval of the intention even when the cue has been recognized [7]. Thus, helping patients strengthen the cue-response association can improve the functions in which ProM may have a role [7]. For example, implementation intention training, a commonly used method to enhance ProM, focuses on strengthening cue-response association by using a combination of 'if' and 'then' in the form of "If I encounter X, then I will do Y" [23]. The individual is asked to repeat this sequence a certain number of times (for example, when I reach the office, I will make a phone call to John). An imagery component may also follow it (for example, apart from rehearsing the 'if' and 'then' statements, imagine performing the task for $30 \mathrm{~s}$ ) [24, 25]. Implementation intention training effectively improves ProM in schizophrenia [24, 25]. The improvement is (a) seen even if the cognitive load of the intervening task increases, (b) sustained after the active intervention is stopped, and (c) observed both in terms of laboratorybased as well as real-life ProM tasks [24, 25]. Other strategies to improve ProM in schizophrenia have also been tried. For example, Tawmley et al. [26] found various compensatory strategies such as using a calendar, making to-do lists, prioritizing tasks, and using routines to automate tasks improve ProM in patients with schizophrenia. Since some of the compensatory strategies, such as using the electronic reminders and diary, assist in bringing the juncture to execute an intention into awareness, they decrease the requirement for active monitoring for cues (to execute the task) in the intervening period of intention formation and realization. Thus, they may prove to be more helpful for patients with significantly compromised cognitive abilities (often observed in patients with chronic illness). However, we need more research to ascertain what type of patients (in terms of 
cognitive abilities, symptom profiles etc.) respond best to a given strategy.

\section{Motivation Enhancement Needs to be Integrated into ProM Remedial Strategies for Schizophrenia}

While attempting to improve ProM in patients with schizophrenia, motivation enhancement must be made an integral part of the remediation program. There are two primary reasons for this argument: (a) amotivation (avolition) is one of the core features of schizophrenia [27], and (b) research findings implicate the role of motivation in the neuropsychological task performance in patients with schizophrenia [28]. Henry et al. [29] found that self-initiated reminders (such as developing own 'to-do' list) are more effective than other reminders (such as automatic text message service that sends out reminders before a target action) in facilitating the execution of intentions in patients with schizophrenia. It suggests the possible role of motivation in executing a task. The person likely develops the 'to-do' list as per his/her priorities. In contrast, automatic reminders may remind the individual about something which may have clinical significance (e.g., meeting the doctor or going for physical exercise); however, the individual may not be interested in executing the task. Let us take an example of the relationship between ProM and medication adherence in schizophrenia. Several studies have shown that ProM impairments correlate with poor medication adherence. However, the relationship may not be perfectly linear as motivation to adhere to medication regimens may act as a significant confounding variable [30]. If the individual is not keen to continue medication, then non-adherence cannot be attributed to ProM impairments even if the individual's performance on a test of ProM is poor. Lam et al.'s [6] research on the role of ProM in medication adherence highlights this complexity. They found that ProM predicted medication adherence in simulated laboratory-based assessment with the help of MMAA; nonetheless, the same was not true in the real-life condition when the actual medication intake over 3 months period was taken into account. Instead, a more complex role of ProM in medication adherence emerged. The predictors for the medication adherence were insight, and the severity of psychopathology while the ProM score improved the predictive power of these variables. The authors opine that ProM may have a significant role when people unintentionally miss taking medications. In contrast, the level of insight could mediate the intentional non-intake of drugs.

Therefore, the ProM remediation strategies such as implementation intention training and the use of checklists and electronic notebooks with reminders should be adjuvanted by other targeted interventions for motivation enhancement. Also, awareness of the ProM deficits and their probable effects on the sociooccupational functioning can significantly influence the patient's motivation to engage in the remedial strategies. Therefore, educating patients about ProM impairments and their effects may enhance motivation to engage in the activities aimed at improving ProM [31].

The foregoing discussion suggests that ProM is crucial for independent living, is impaired in patients with schizophrenia and the impairment is associated with poor functioning. We believe that it is now time that ProM remediation is made an integral part of the rehabilitation process of patients with schizophrenia. While attempting ProM remedial strategies, factors such as the patient's motivation to execute the tasks and the awareness about the effects of impaired ProM on everyday functioning should be addressed through motivation enhancement interventions and educating patients about the role ProM plays in our everyday life.

\section{References}

1. Dalla Barba G. Prospective memory: A new memory system? In: Boller F, Grafman J, editors. Handbook of neuropsychology. New York: Elsevier; 1993. p. 239-51.

2. McDaniel MA, Einstein GO. Strategic and automatic processes in prospective memory retrieval: a multiprocess framework. Appl Cognit Psychol. 2000;14:S127-44.

3. Gardner RS, Ascoli GA. The natural frequency of human prospective memory increases with age. Psychol Aging. 2015;30:209-19.

4. Kumar D. Prospective memory: the memory for future intentions. Indian J Clin Psychol. 2018;45:18-24.

5. Brandimonte MA, Ferrante D. The social side of prospective memory. Prospective memory: cognitive, neuroscience, developmental, and applied perspectives. New York: Lawrence Erlbaum; 2008. p. 347-65.

6. Lam JW, Lui SS, Wang Y, Chan RC, Cheung EF. Prospective memory predicts medication management ability and correlates with non-adherence to medications in 
individuals with clinically stable schizophrenia. Schizophr Res. 2013;147:293-300.

7. Raskin SA, Maye J, Rogers A, Correll D, Zamroziewicz M, Kurtz M. Prospective memory in schizophrenia: relationship to medication management skills, neurocognition, and symptoms in individuals with schizophrenia. Neuropsychology. 2014;28:359-65.

8. Burgess PW, Scott SK, Frith CD. The role of the rostral frontal cortex (area 10) in prospective memory: a lateral versus medial dissociation. Neuropsychologia. 2003;41:906-18.

9. Massa F, Grisanti S, Brugnolo A, Doglione E, Orso B, Morbelli S, et al. The role of anterior prefrontal cortex in prospective memory: an exploratory FDG-PET study in early Alzheimer's disease. Neurobiol Aging. 2020;96:117-27.

10. Wong AHC, Van Tol HH. Schizophrenia: from phenomenology to neurobiology. Neurosci Biobehav Rev. 2003;27:269-306.

11. Reichenberg A, Harvey PD. Neuropsychological impairments in schizophrenia: integration of performance-based and brain imaging findings. Psychol Bull. 2007;133:833-58.

12. Kumar D, Nizamie SH, Jahan M. Event-based prospective memory in schizophrenia. J Clin Exp Neuropsychol. 2005;27:867-72.

13. Lui SS, Wang Y, Liu AC, Chui WW, Gong Q, Shum D, et al. Prospective memory in patients with first-onset schizophrenia and their non-psychotic siblings. Neuropsychologia. 2011;49:2217-24.

14. Ordemann GJ, Opper J, Davalos D. Prospective memory in schizophrenia: a review. Schizophr Res. 2014;155:77-89.

15. Kumar D, Nizamie SH, Jahan M. Activity-based prospective memory in schizophrenia. Clin Neuropsychol. 2008;22:497-506.

16. Zhuo K, Lu Y, Yang Z, Fan X, Song Z, Liao L, et al. Prospective memory performance in patients with drugnaïve, first-episode psychosis. Schizophr Res. 2013;143:285-90.

17. Henry JD, Rendell PG, Kliegel M, Altgassen M. Prospective memory in schizophrenia: Primary or secondary impairment? Schizophr Res. 2007;95:179-85.

18. Saleem S, Kumar D, Venkatasubramanian G. Prospective memory in first-degree relatives of patients with schizophrenia. Clin Neuropsychol. 2018;32:993-1001.

19 Zhou F-C, Hou W-M, Wang C-Y, Ungvari GS, Chiu HF, Correll CU, et al. Prospective memory performance in nonpsychotic first-degree relatives of patients with schizophrenia: a controlled study. PLoS ONE. 2014;9:e111562.

20. Twamley EW, Woods SP, Zurhellen $\mathrm{CH}$, Vertinski M, Narvaez JM, Mausbach BT, et al. Neuropsychological substrates and everyday functioning implications of prospective memory impairment in schizophrenia. Schizophr Res. 2008; 106:42-9.

21. Au RW, Man D, Xiang Y-T, Shum D, Lee E, Ungvari GS, et al. Prospective memory predicts the level of community living skills in schizophrenia. Psychiatry Res. 2014;219:86-91.

22. Patterson TL, Lacro J, McKibbin CL, Moscona S, Hughs T, Jeste DV. Medication management ability assessment: results from a performance-based measure in older outpatients with schizophrenia. J Clin Psychopharmacol. 2002;22:11-9.

23. Gollwitzer PM. Implementation intentions: strong effects of simple plans. Am Psychol. 1999;54:493-503.

24. Chen T, Liu L, Cui J, Li Y, Qin X, Tao S, et al. Implementation intention training for prospective memory in schizophrenia: a 3-month follow-up study. Schizophr Res. 2019;206:378-85.

25. Chen X-J, Liu L-L, Cui J-F, Gan M-Y, Li C-Q, Neumann DL, et al. The effect and mechanisms of implementation intention in improving prospective memory performance in schizophrenia patients. Psychiatry Res. 2016;244:86-93.

26. Twamley EW, Vella L, Burton CZ, Heaton RK, Jeste DV. Compensatory cognitive training for psychosis: effects in a randomized controlled trial. J Clin Psychiatry. 2012;73:1212-9.

27. Foussias G, Remington G. Negative symptoms in schizophrenia: avolition and Occam's razor. Schizophr Bull. 2010;36:359-69.

28 Moritz S, Silverstein SM, Beblo T, Özaslan Z, Zink M, Gallinat J. Much of the neurocognitive impairment in schizophrenia is due to factors other than schizophrenia itself: implications for research and treatment. Schizophr Bull Open. 2021;2:sgaa034.

29. Henry JD, Moore P, Terrett G, Rendell PG, Scott JG. A comparison of different types of prospective memory reminders in schizophrenia. Schizophr Res. 2019;210:89-93.

30. Zogg JB, Woods SP, Sauceda JA, Wiebe JS, Simoni JM. The role of prospective memory in medication adherence: a review of an emerging literature. $\mathrm{J}$ Behav Med. 2012;35:47-62.

31. Wang Y, Chan RC, Shum DH. Schizophrenia and prospective memory impairments: a review. Clin Neuropsychol. 2018;32:836-57.

Publisher's Note Springer Nature remains neutral with regard to jurisdictional claims in published maps and institutional affiliations. 